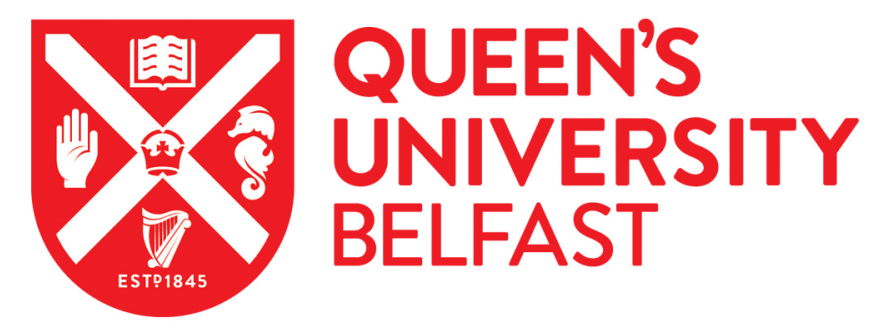

\title{
Absolute Calibration of Atomic Density Measurements by Laser- Induced Fluorescence Spectroscopy with Two-Photon Excitation
} (TALIF)

Niemi, K., Schulz-von Der Gathen, V., \& Döbele, H. F. (2001). Absolute Calibration of Atomic Density Measurements by Laser-Induced Fluorescence Spectroscopy with Two-Photon Excitation (TALIF). Journal of Physics D: Applied Physics, 34(15), 2330-2335. https://doi.org/10.1088/0022-3727/34/15/312

\section{Published in:}

Journal of Physics D: Applied Physics

Queen's University Belfast - Research Portal:

Link to publication record in Queen's University Belfast Research Portal

\section{General rights}

Copyright for the publications made accessible via the Queen's University Belfast Research Portal is retained by the author(s) and / or other copyright owners and it is a condition of accessing these publications that users recognise and abide by the legal requirements associated with these rights.

Take down policy

The Research Portal is Queen's institutional repository that provides access to Queen's research output. Every effort has been made to ensure that content in the Research Portal does not infringe any person's rights, or applicable UK laws. If you discover content in the Research Portal that you believe breaches copyright or violates any law, please contact openaccess@qub.ac.uk. 


\title{
Absolute calibration of atomic density measurements by laser-induced fluorescence spectroscopy with two-photon excitation
}

\author{
K Niemi, V Schulz-von der Gathen and H F Döbele \\ Institut für Laser- und Plasmaphysik, Universität Essen, D-45117 Essen, Germany \\ E-mail:svdg@uni-essen.de \\ Received 11 April 2001 \\ Published 17 July 2001 \\ Online at stacks.iop.org/JPhysD/34/2330
}

\begin{abstract}
The two-photon resonances of atomic hydrogen $(\lambda=2 \times 205.1 \mathrm{~nm})$, atomic nitrogen $(\lambda=2 \times 206.6 \mathrm{~nm})$ and atomic oxygen $(\lambda=2 \times 225.6 \mathrm{~nm})$ are investigated together with two selected transitions in krypton $(\lambda=2 \times 204.2 \mathrm{~nm})$ and xenon $(\lambda=2 \times 225.5 \mathrm{~nm})$. The natural lifetimes of the excited states, quenching coefficients for the most important collisions partners, and the relevant ratios of the two-photon excitation cross sections are measured. These data can be applied to provide a calibration for two-photon laser-induced fluorescence measurements based on comparisons with spectrally neighbouring noble gas resonances.
\end{abstract}

\section{Introduction}

Laser-induced fluorescence (LIF) spectroscopy is an optical diagnostic method that yields space and time resolved populations, in particular of atomic ground states in plasmas [1]. Ground-state atoms are excited resonantly into a higher electronic states. The subsequent fluorescence permits one, in principle, to infer the ground-state population. Absolute densities can be determined if a suitable method of calibration is available. Collisional quenching processes leading to an additional radiationless de-excitation of the induced population have to be included.

Single-photon LIF methods can usually be calibrated by Rayleigh scattering. A reference gas-usually a noble gaswith a known Rayleigh scattering cross section is filled into the experimental chamber. The Rayleigh scattered signal is observed under identical conditions for the excitation and detection and can, therefore, provide a reference [2]. The excitation wavelengths of light atoms are located, however, in the vacuum ultraviolet (VUV) spectral range and are, therefore, only accessible with considerable experimental effort. Other problems are the strong absorption of the VUV radiation by many gases and even by the medium under investigation at elevated densities (optical thickness).
These problems can be circumvented by two-photon laserinduced fluorescence (TALIF) spectroscopy. The two-photon excitation is, however, much less efficient than the singlephoton excitation, so that fluorescence signals can be obtained in most cases only with focused laser radiation. This entails good spatial resolution which is generally determined by the dimensions of the focus. The nonlinear character of the two-photon excitation process excludes the applicability of Rayleigh calibration. The generally applied calibration uses reference sources that are able to provide specified densities of the species under investigation, for example flow tubes with microwave excitation in combination with titration methods [3]. This necessitates, however, that the reference source can be incorporated in the experimental set-up and is compatible with the vacuum requirements.

The objective of this contribution is to provide a calibration method for TALIF diagnostics to determine ground-state densities of atomic species such as hydrogen, nitrogen, and oxygen in low-temperature plasmas, including collisional effects. The proposed method is based on a comparative measurement at a spectrally close two-photon resonance of a noble gas [4]. This method is independent of other diagnostics and needs no major experimental modifications. The well known two-photon resonances of atomic hydrogen, nitrogen, and oxygen have corresponding transitions in krypton and 


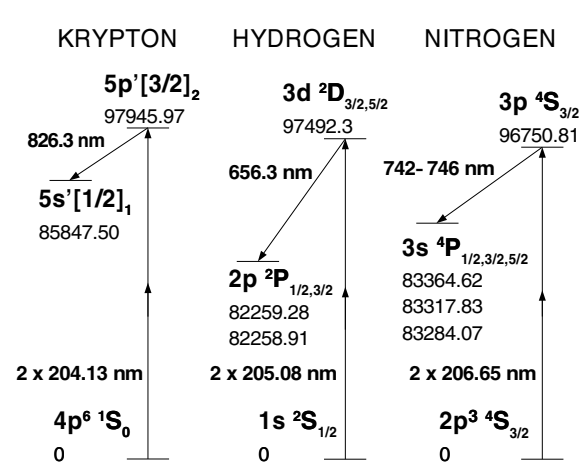

Figure 1. Two-photon excitation schemata of krypton, hydrogen, and nitrogen. The level energies are given in $\mathrm{cm}^{-1}$.

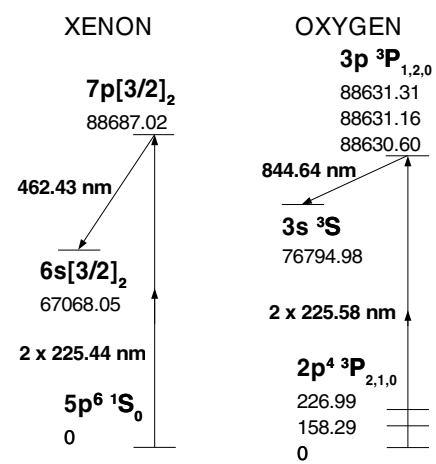

Figure 2. Two-photon excitation schemata of xenon and oxygen. The level energies are given in $\mathrm{cm}^{-1}$.

xenon. We have investigated these transitions (shown in figures 1 and 2) by two-photon excitation and have measured the atomic data necessary for calibration: natural lifetimes of excited states and quenching coefficients for the most important collision partners, as well as the relevant ratios of the two-photon cross sections.

\section{Principle of the calibration method}

The calibration is based on a reference measurement with a noble gas of known concentration. A prerequisite for its applicability is that both TALIF excitations are performed with identical spatial, spectral, and temporal intensity distribution of the laser radiation. This removes the necessity to know the intensity distribution explicitly. The condition is best fulfilled if the two-photon resonances are spectrally as close as possible. It is also necessary that the signal intensity as a function of laser energy obeys the same scaling law for both measurements. It is therefore only possible to compare measurements where the excitation is not influenced by saturation. We shall describe the signal intensity in the following for the case of an unsaturated two-photon excitation using a simple rate equation system. In the case of excitation with sufficiently low laser intensities a depletion of the ground-state population $n_{1}(t) \approx n_{0}$ as well as photo-ionization effects on the excited-state population $n_{2}(t)$ can be neglected:

$$
\frac{\mathrm{d}}{\mathrm{d} t} n_{2}(t)=R(t) n_{1}(t)-A_{2} n_{2}(t)
$$

$$
\frac{\mathrm{d}}{\mathrm{d} t} n_{1}(t)=-R(t) n_{1}(t) .
$$

The effective de-excitation rate $A_{2}=\tau^{-1}+Q$ of the excited state comprises the spontaneous emission with natural lifetime $\tau$ and the radiationless quenching with the effective quenching rate $Q$. The two-photon excitation rate $R(t)$ can be expressed by the generalized excitation cross section $\sigma^{(2)}$ :

$$
R(t)=G^{(2)} \sigma^{(2)} g(\Delta v)\left(\frac{I_{0}(t)}{h v}\right)^{2} .
$$

The normalized line profile $g(\Delta v)$ of the two-photon excitation $\left(\int g(v) \mathrm{d} v=1\right)$ is composed of the convolution of the one-photon spectral profile $g_{L}(v)$ with the central frequency $v_{L}$ and the absorption line of the atom $g_{A}(v)$ with the two-photon resonance frequency $v_{A}$ according to

$g\left(\Delta v=2 v_{L}-v_{A}\right)=g_{A}\left(2 v-v_{A}\right) * g_{L}\left(v-v_{L}\right) * g_{L}\left(v-v_{L}\right)$.

The photon statistic factor $G^{(2)}$ has the value 2 [5] for the absorption of two photons from a chaotic radiation field of a multi-mode laser (averaged over many coherence times of the fluctuating phase). Integrating the rate equation (1) leads to

$$
\begin{gathered}
n_{1}(t)=n_{0} \exp \left(-\int_{0}^{t} R\left(t^{\prime}\right) \mathrm{d} t^{\prime}\right) \approx n_{0} \\
n_{2}(t)=n_{0} \int_{0}^{t} R\left(t^{\prime}\right) \mathrm{e}^{-A_{2}\left(t-t^{\prime}\right)} \mathrm{d} t^{\prime} .
\end{gathered}
$$

The number of photons per unit volume in the fluorescence channel to an intermediate state labelled 3 is given by $\left(a_{23}=\right.$ $A_{23} / A_{2}$ is the optical branching ratio)

$n_{F}=A_{23} \int_{0}^{\infty} n_{2}(t) \mathrm{d} t=n_{0} a_{23} \frac{G^{(2)} \sigma^{(2)}}{(h v)^{2}} g(\Delta v) \int_{0}^{\infty} I_{0}^{2}(t) \mathrm{d} t$.

The time and spectrally integrated photomultiplier signal $S_{P M T}$ results by integration of $n_{F}$ over the two-photon resonance and the observed excitation volume taking into account the observation sensitivity:

$$
S_{P M T}=\frac{\Delta \Omega}{4 \pi} T \eta G e_{0} R_{L} \iint n_{F}(\vec{r}) \mathrm{d} V \mathrm{~d} \nu .
$$

Here $\Delta \Omega$ stands for the solid angle of detection; $T$ and $\eta$ are the transmission of the observation optics and the quantum efficiency of the photomultiplier at the fluorescence wavelength, respectively; $G$ is the amplification of the photomultiplier; $R_{L}$ is the load resistor; and $e_{0}$ is the elementary charge.

We introduce the normalized fluorescence signal

$$
I_{F}=\frac{S_{P M T}}{\iint I_{0}^{2}(t, \vec{r}) \mathrm{d} t \mathrm{~d} V}
$$

and obtain from equation (6) for a reference gas $\mathrm{R}$ and an atomic species $\mathrm{X}$ the following expression for the atomic density

$$
n_{X}=\gamma \frac{a_{23}(\mathrm{R})}{a_{23}(\mathrm{X})} \frac{\sigma^{(2)}(\mathrm{R})}{\sigma^{(2)}(\mathrm{X})} \frac{I_{F}(\mathrm{X})}{I_{F}(\mathrm{R})} n_{R}=\chi \frac{I_{F}(\mathrm{X})}{I_{F}(\mathrm{R})} n_{R} .
$$




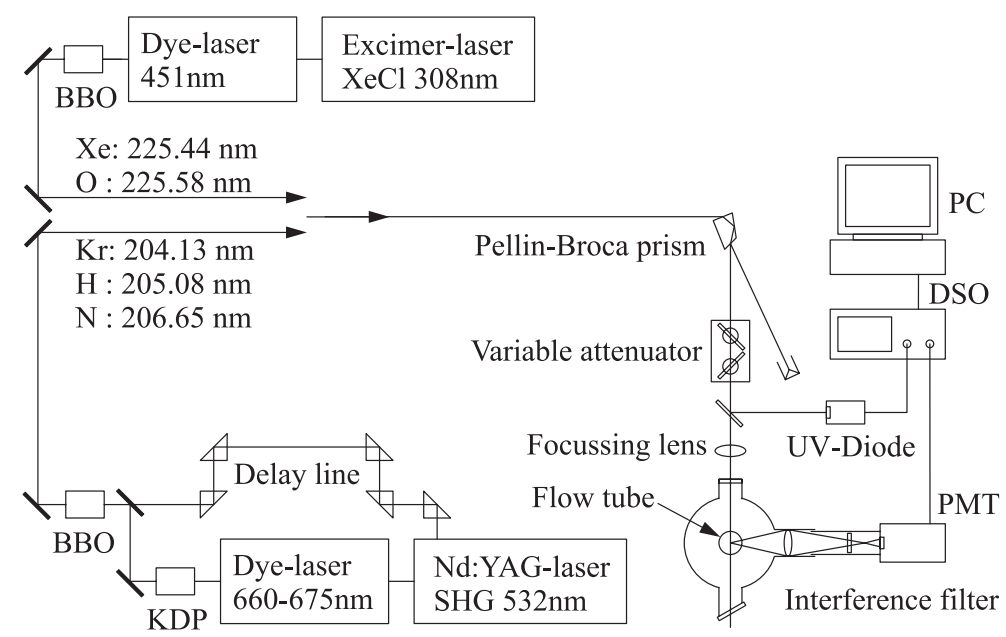

Figure 3. Experimental set-up for the TALIF measurements.

In this equation $\gamma$ describes the ratio of the detection sensitivities for the observed fluorescence wavelengths. Apart from the quenching rates, this factor $\chi$ is composed only of atomic and experimental constants.

The purely optical branching ratios can be corrected for the collision-dominated case (where a time resolved measurement of the fluorescence is no longer possible), if the concentrations and the quenching coefficients of the effective collision partners are known. The ratio of the two-photon cross sections can be determined by comparison of two measurements with known densities. The atomic density according to equation (8) can, therefore, be determined independently of explicit knowledge of the laser intensity distribution by a comparative measurement with a known noble gas density.

We describe, for the sake of completeness, the saturation effects occurring with increasing laser intensity. The increasing depletion of the ground state leads to a reduction of the signal as in the case of single-photon excitation. Photoionization out of the excited state by absorption of a third photon represents an efficient loss channel out of the system of the levels considered, so that a decrease of the signal amplitude results [6]. Amplified stimulated emission (ASE) is also a possibility, if at higher atomic densities the fluorescence transition exhibits a population inversion. This radiation is then preferentially emitted in the direction of the exciting laser beam. A complete calculation of these processes in the interaction volume on the basis of rate and transport equations [1] depends sensitively on the cross sections and beam parameters which are only insufficiently known in most cases.

\section{Experiment}

\subsection{Two-photon excitation set-up}

The TALIF experiment is performed in a vacuum chamber that can accept a so-called flow-tube reactor as described in the next section. Figure 3 shows the basics of the setup. Pump radiation at $\lambda=205 \mathrm{~nm}$ is generated with the aid of a dye laser system (Spectron SL400) pumped with a second harmonic $(\lambda=532 \mathrm{~nm})$ of a Nd:YAG laser
(Spectron SL800) by mixing the frequency-doubled dye laser output and the frequency-doubled $\mathrm{Nd}$ :YAG radiation in a BBO-crystal. The dye is a mixture of Pyridine 1 and DCM in propylene carbonate. The system provides a maximum UV energy of $0.5 \mathrm{~mJ}$ in $8 \mathrm{~ns}$ with a repetition rate of $10 \mathrm{~Hz}$. Pump radiation at $\lambda=225 \mathrm{~nm}$ is generated with a $\mathrm{XeCl}$ excimer laser (Lambda Physik EMG200E) which pumps a dye laser (Radiant Dyes LDL205) with a frequency-doubling unit. The dye is a mixture of Coumarin 47 and Coumarin 120, dissolved in methanol. Up to $2 \mathrm{~mJ}$ with a pulse duration of $10 \mathrm{~ns}$ and a repetition rate of $2 \mathrm{~Hz}$ are obtained. The UV radiation is singled-out with a Pellin-Broca prism and is focused with an $f=30 \mathrm{~cm}$ suprasil lens into the vacuum vessel. Fluorescence radiation is observed transverse to the laser beam and is detected with a Hamamatsu R928 or Burle C31034A photomultiplier equipped with interference filters. The laser energy is monitored with a fast UV diode. The fluorescence signal and the reference signal of this fast diode are displayed simultaneously with a digital oscilloscope and are stored in a computer. A variable attenuator was inserted into the UV beam in order to analyse the intensity dependence and to exclude saturation. This attenuator consists of two dielectrically coated suprasil substrates that can be inclined which respect to each other in order to avoid lateral displacements of the beam and focus. The attenuation is due to the angular dependence of the reflection properties of the dielectric coating.

The excitation wavelengths of hydrogen, nitrogen, and krypton do not coincide as ideal as in case of oxygen and xenon. Nevertheless we assume the same excitation conditions also in case of the first three species for the following arguments. The conditions of generation and amplification of the dye laser radiation are comparable, since the relevant wavelengths are located in the central part of the tuning range of the dye mixture. Optogalvanic measurements of various Dopplerbroadened Ne-lines indicate that the spectral profile of the dye laser is obviously unchanged. The same is true for the temporal behaviour of the UV laser pulses. No changes could be measured. 


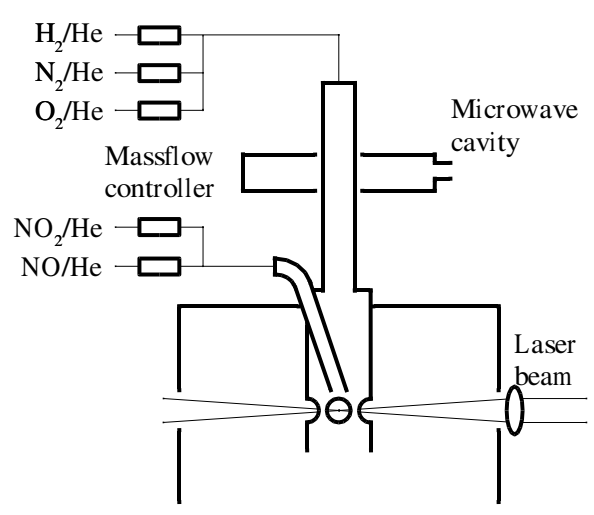

Figure 4. Flow-tube reactor incorporated into the experimental set-up.

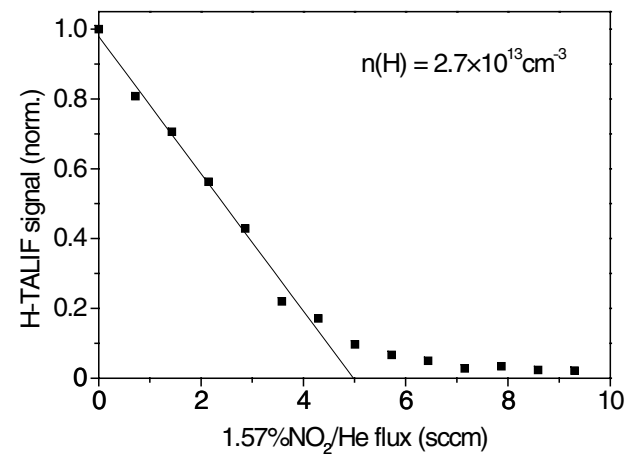

Figure 5. Titration curve for hydrogen $\left(705 \mathrm{sccm}\right.$ of $7.1 \% \mathrm{H}_{2} / \mathrm{He}$ at $990 \mathrm{~Pa}$ and a microwave power of $100 \mathrm{~W}$ ).

\subsection{Flow-tube reactor}

A flow-tube reactor with microwave excitation serves as a source of the atomic species. Figure 4 explains the combination of the flow-tube reactor with the experiment. The molecular gases dissolved in helium are admitted coaxially to the cylindrical resonator of a microwave discharge $(2.45 \mathrm{GHz})$ in which the atoms are generated. The discharge is connected with a Teflon tube leading to the centre of the vacuum chamber. Four holes in the flow-tube provide the access for the TALIF measurements. The absolute value of the atomic density in the observation volume is established by a titration method: reactive titration gas is admitted to the main flow upstream of the observation volume. A fraction of the atoms is destroyed in fast reactions according to

$$
\begin{array}{cc}
\mathrm{H}+\mathrm{NO}_{2} \rightarrow \mathrm{OH}+\mathrm{NO} & k=1.3 \times 10^{-10} \mathrm{~cm}^{3} \mathrm{~s}^{-1} \\
\mathrm{~N}+\mathrm{NO} \rightarrow \mathrm{N}_{2}+\mathrm{O} & k=2 \times 10^{-11} \mathrm{~cm}^{3} \mathrm{~s}^{-1} \\
\mathrm{O}+\mathrm{NO}_{2} \rightarrow \mathrm{NO}+\mathrm{O}_{2} & k=9.5 \times 10^{-12} \mathrm{~cm}^{3} \mathrm{~s}^{-1}
\end{array}
$$

where $k$ stands for the reaction constant for $T=300 \mathrm{~K}$ from [3]. If the reaction path is complete, each reactive molecule leads to the destruction of one atom. A plot of the measured TALIF signal against the titration flux (figure 5) is expected to exhibit a linear decay. The extrapolation to the point of zero signal amplitude defines the point of titration at which the flux $\phi_{T}$ of the reactive molecules equals the flux

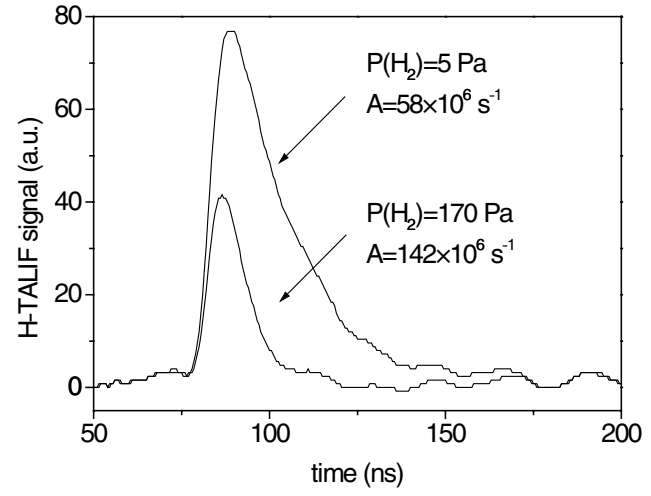

Figure 6. Time resolved $\mathrm{H}\left(3 \mathrm{~d}^{2} \mathrm{D}_{J}\right)$ TALIF signal for different $\mathrm{H}_{2}$ pressures.

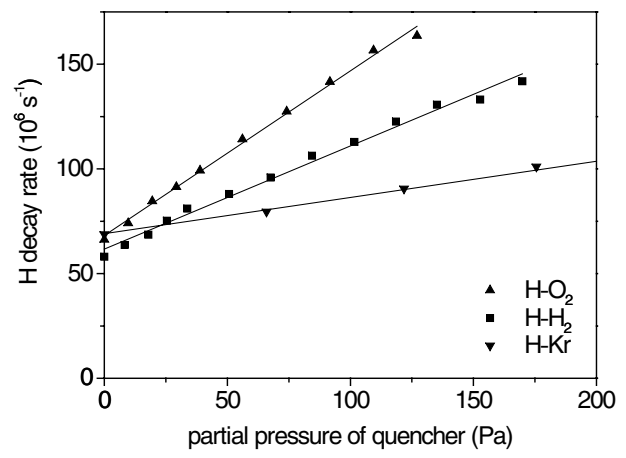

Figure 7. Stern-Volmer plots: de-excitation rate of the $\mathrm{H}\left(3 \mathrm{~d}^{2} \mathrm{D}_{J}\right)$ TALIF signal against the partial pressure of molecular oxygen, molecular hydrogen and krypton.

of the atoms. The atomic density is obtained from the total flux $\phi_{T \text { ОT }}$, the pressure $p$, and the gas temperature $T$ according to

$$
n_{A}=\frac{p}{k T} \frac{\phi_{T}}{\phi_{T O T}} .
$$

The inert carrier gas helium leads to a more efficient dissociation and stabilizes the discharge conditions induced by the titration flux.

All gases are admitted in a controlled manner with mass flow controllers. The total pressure is determined with a capacitive pressure gauge working independently of the kind of gas used. The gas temperature was measured to be $T=300 \mathrm{~K}$ from the population distribution of the fine structure-split ground state of the oxygen atoms $\left(2 \mathrm{p}^{4}{ }^{3} \mathrm{P}_{J}, J=2,1,0\right)$; the fine-structure component $2 \mathrm{p}^{4}{ }^{3} \mathrm{P}_{2}$ was determined to be $74 \%$. The contents of $\mathrm{NO}$ and $\mathrm{NO}_{2}$ in the titration gas mixtures were determined with a FTIR spectrometer. Atomic hydrogen and atomic nitrogen densities were generated in the range of $10^{13} \mathrm{~cm}^{-3}$ and atomic oxygen densities in the range of $10^{14} \mathrm{~cm}^{-3}$ under typical conditions (microwave power $P=$ $\left.100 \mathrm{~W}, p=1000 \mathrm{~Pa}, \phi_{T O T}=700 \mathrm{sccm}, \phi_{H_{2}, N_{2}, O_{2}}=5 \mathrm{sccm}\right)$. 
Table 1. Natural lifetimes $\tau$ and quenching coefficients $k_{q}$ of the investigated states for $T=300 \mathrm{~K}$.

\begin{tabular}{llcccccccc}
\hline & & \multicolumn{7}{c}{$\mathrm{k}_{q}\left(10^{-10} \mathrm{~cm}^{3} \mathrm{~s}^{-1}\right)$ for reagent } \\
\cline { 3 - 10 } Excited state & $(\mathrm{ns})$ & \multicolumn{1}{c}{$\mathrm{H}_{2}$} & \multicolumn{1}{c}{$\mathrm{N}_{2}$} & \multicolumn{1}{c}{$\mathrm{O}_{2}$} & $\mathrm{He}$ & $\mathrm{Ar}$ & $\mathrm{Kr}$ & $\mathrm{Xe}$ & $\mathrm{CH}_{4}$ \\
\hline $\mathrm{Kr}\left(5 \mathrm{p}^{\prime}[3 / 2]_{2}\right)$ & 34.1 & 8.44 & \multicolumn{1}{c}{3.35} & 6.34 & 0.78 & 1.29 & 1.46 & 3.78 & 6.27 \\
$\mathrm{H}\left(3 \mathrm{~d}^{2} \mathrm{D}_{J}\right)$ & 17.6 & 20.4 & 20.1 & 32.6 & 0.18 & 3.93 & 7.15 & 19.8 & 25.0 \\
$\mathrm{~N}\left(3 \mathrm{~s}^{4} \mathrm{P}_{3 / 2}\right)$ & 29.6 & 1.11 & 0.41 & 6.63 & 0.11 & 0.37 & 3.16 & 7.75 & 5.81 \\
\hline $\mathrm{Xe}\left(7 \mathrm{p}[3 / 2]_{2}\right)$ & 105.6 & 28.5 & 14.0 & 20.6 & 2.33 & 3.71 & 5.91 & 5.05 & - \\
$\mathrm{O}\left(3 \mathrm{p}^{3} \mathrm{P}_{J}\right)$ & 35.1 & 10.9 & 5.9 & 9.3 & 0.07 & 0.25 & - & - & - \\
\hline
\end{tabular}

\section{Measurements and results}

\subsection{Natural lifetimes and quenching coefficients}

Variable fluxes of a number of quenching gases have been applied to the flow-tube reactor through the titration tube in order to determine the natural lifetime and the quenching coefficient of an excited state. The decay of the excited state after excitation by laser radiation is investigated by time resolved measurement of its fluorescence radiation (figure 6). The decay rate of the TALIF signal is determined on the basis of an exponential fit; it is displayed as a function of the partial pressure of the quenching gas in figure 7 . In this so-called Stern-Volmer plot a linear pressure dependence of the decay rate $A_{i}$ of an excited state $i$ is expected

$$
A_{i}=\frac{1}{\tau_{i}}+\sum_{q} k_{q}^{i} n_{q}
$$

with $\tau_{i}$ as the natural lifetime, $n_{q}$ as the density of the collision partner $q$, and $k_{q}^{i}$ as the specific quenching coefficient. The slope of the curve allows one to determine a quenching coefficient which can be displayed in units of $\mathrm{cm}^{3} \mathrm{~s}^{-1}$ with the ideal gas law using the measured gas temperature of $T=300 \mathrm{~K}$. It is worth noting that quenching coefficients have usually a non-trivial temperature dependence [7] apart from this unit conversion. The natural lifetime is determined from the crossing point of the curve with the $Y$-axis. The effective quenching rate of the molecular fraction and carrier gas is included in case of an atomic species; in the case of a noble gas the same is true for the self-quenching rate.

The natural lifetimes of the excited states and the quenching coefficients of the gases $\mathrm{H}_{2}, \mathrm{~N}_{2}, \mathrm{O}_{2}, \mathrm{He}$, Ar, Xe, $\mathrm{Kr}$, and $\mathrm{CH}_{4}$ were determined. It is not possible, however, to generate sufficiently high atomic densities in the flow-tube reactor to investigate the self-quenching of the atomic species [8]. The pressure of the quenching gas was increased stepwise until it was no longer possible to resolve the fluorescence signal in time. The maximum time resolution of approximately $7 \mathrm{~ns}$ is mainly determined by the laser pulse duration rather than by the response of the detection system (photomultiplier tube, digital storage oscilloscope). No systematic deviations from a linear pressure dependence were observed in this range. The resulting values that we consider to be correct within an error of approximately $10 \%$ are summarized in table 1 ; they will be compared in the following with published data.

For the hydrogen state we find a satisfactory agreement with values from $[9,10]$. The values in our experiment for oxygen are systematically higher by approximately $30 \%$ than those communicated in [9]. We find again good agreement for $\tau_{X e}, k_{X e}^{X e}$, and $k_{A r}^{X e}$ with values from [11]; the values for $k_{H e}^{X e}$, and $k_{K r}^{X e}$ are higher by a factor of $\approx 1.7$, however. The value for $k_{A r}^{K r}$ is in agreement with that measured in [12]; our value for $\tau_{K r}=34.1 \mathrm{~ns}$ exceeds the value of $26.9 \mathrm{~ns}$ which was given in [12]. A possible reason could be the neglect of the self-quenching rate of the krypton state in the earlier work. Our value for $\tau_{N}$ is in satisfactory agreement with the corresponding value [13], our value of $k_{N_{2}}^{N}=0.41 \times 10^{-10} \mathrm{~cm}^{3} \mathrm{~s}^{-1}$ is smaller than the value from the reference of $0.67 \times 10^{-10} \mathrm{~cm}^{3} \mathrm{~s}^{-1}$. For the other quenching coefficients we had no basis of comparison.

Some qualitative trends can be seen in table 1 . The coefficient for the quenching reaction of one excited species increases with the mass of a noble gas collider. This indicates a transfer reaction, which becomes effective with an increasing number of states able to accept at least part of the excitation energy. According to this argument the measured quenching coefficients for reactions with the molecules are larger, except for the excited nitrogen state. This discrepancy can be explained by reactive quenching which is excepted to be very effective in particular for excited noble gas atoms, for example excimer formation. A more comprehensive treatment of the quenching processes can be found in [26].

The excited hydrogen state $n=3$ deserves a more detailed discussion. Since the energy spread of the fine-structure levels $3 \mathrm{~s}, 3 \mathrm{p}$, and $3 \mathrm{~d}$ is smaller than the laser bandwidth, both the $3 \mathrm{~s}$ and the $3 \mathrm{~d}$ states are populated from the $1 \mathrm{~s}$ ground state according to the selection rules for two-photon transitions. We have measured the natural lifetime $\tau_{H}=17.6 \mathrm{~ns}$. This is consistent with the theoretical values of the natural lifetimes $\tau(3 s)=159 \mathrm{~ns}$ and $\tau(3 d)=15.6 \mathrm{~ns}$ according to [14] and the ratio of the excitation cross sections $\sigma^{(2)}(3 d) / \sigma^{(2)}(3 s)=7.56$ (see [15]). We cannot conclude from our measurements at higher pressures, whether the collisional de-excitation of the $3 \mathrm{~s}$ and the $3 \mathrm{~d}$ states occurs in a radiationless manner or via a population transfer in the $3 \mathrm{p}$ state whose optical de-excitation in $\mathrm{H}_{\alpha}$ and $\mathrm{L}_{\alpha}$ transitions (to $2 \mathrm{~s}$ and 1s) is partly included in the detection. This so-called state mixing is considered in $[10,16]$ where the state mixing is proportional to the pressure and is described by specific rate coefficients. The communicated rate coefficients are in considerable disagreement; in [17] complete state mixing is observed at, clearly, lower pressures.

\subsection{Two-photon excitation cross sections}

Two-photon excitation cross sections of atomic hydrogen $[15,18]$, oxygen $[19,20]$ and nitrogen [21] have been theoretically determined. Only the oxygen cross section has been verified experimentally [22], to the best of our knowledge. 


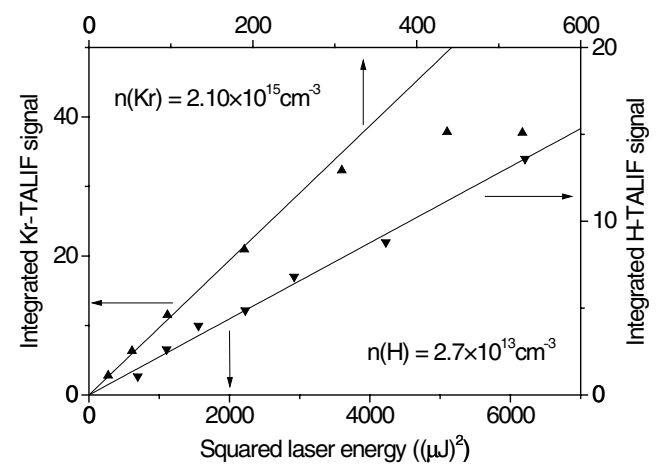

Figure 8. Integrated (see text) $\operatorname{Kr}\left(5 \mathrm{p}^{\prime}[3 / 2]_{2}\right)$ and $\mathrm{H}\left(3 \mathrm{~d}^{2} \mathrm{D}_{J}\right)$ TALIF signals as functions of the squared laser energy.

This was the basis for the determination of the xenon cross section [4]. No data exist, to the best of our knowledge, for the krypton cross section. We have therefore determined the ratios of the two-photon cross sections in this work.

We generate a stationary atomic density in the flow-tube reactor with the aid of the titration method. The subsequent fluorescence radiation, integrated spectrally over the twophoton resonance, is measured as a function of the laser energy and is time integrated. The reference gas is then admitted to the previously evacuated vacuum vessel at a pressure of several pascals, and the measurement procedure is repeated.

The integrated TALIF signals are plotted, as shown in figure 8, against the square of the laser energy. The measured signal points following, for small energy, the quadratic dependence of a non-saturated two-photon excitation are fitted by a line through the origin. The ratio of the slopes of these lines corresponds to the ratio of the normalized fluorescence signals. The calibration factor $\chi$ results from equation (8) on the basis of the known densities.

The ratio of the detection sensitivities for the fluorescence wavelength (at constant photomultiplier tube voltage) is determined from manufacturer's data of the photomultiplier quantum efficiency and measured relative transmissions of the interference filters. Whereas the fluorescence light is emitted in only one transition in the case of the atomic species, several fluorescence channels exist for the noble gas states: $\mathrm{Xe} 7 \mathrm{p}[3 / 2]_{2} \rightarrow 7 \mathrm{~s}, 6 \mathrm{~s}, 6 \mathrm{~s}^{\prime}, 5 \mathrm{~d}$ and $\mathrm{Kr} 5 \mathrm{p}^{\prime}[3 / 2]_{2} \rightarrow 5 \mathrm{~s}$, $5 s^{\prime}$. Therefore, the effective branching ratios involve, besides the measured natural lifetimes and the quenching coefficients, the purely optical branching ratio $A_{23} / A_{2}=0.958$ of the $826 \mathrm{~nm}$ krypton line (averaged) from [12] and $A_{23}=2.18 \times$ $10^{6} \mathrm{~s}^{-1}$ of the $462 \mathrm{~nm}$ xenon line [23]. The resulting twophoton excitation cross sections are summarized as:

$$
\begin{gathered}
\frac{\sigma^{(2)}(\mathrm{Kr})}{\sigma^{(2)}(\mathrm{H})}=0.62 \quad \frac{\sigma^{(2)}(\mathrm{Kr})}{\sigma^{(2)}(\mathrm{N})}=0.67 \\
\frac{\sigma^{(2)}(\mathrm{Xe})}{\sigma^{(2)}(\mathrm{O})}=0.36
\end{gathered}
$$

We estimate these values to be correct to $50 \%$ in view of the various input parameters and the reproducibility of the laser beam profile. Our value for $\sigma^{(2)}(\mathrm{Xe}) / \sigma^{(2)}(\mathrm{O})$ is well within this limit if compared with the value of 0.51 determined in [4]. In our experiments the fluorescence radiation was observed in the direction parallel to the direction of the polarization of the exciting radiation. In [4] it has been established that the fluorescence radiation of the excited xenon and oxygen atoms is independent of the direction of the polarization. The calibration technique has recently been applied successfully on the basis of our values for $\sigma^{(2)}(K r) / \sigma^{(2)}(N)$ in [24] and for $\sigma^{(2)}(K r) / \sigma^{(2)}(H)$ in [25].

\section{Acknowledgments}

This work was funded by the Deutsche Forschungsgemeinschaft in the frame of the Sonderforschungsbereich 191 and of the Graduiertenkolleg 'Hochtemperatur-Plasmaphysik'. We thank C Fischer and J Leistikow for skilful technical assistance.

\section{References}

[1] Amorim J, Baravian G and Jolly J 2000 J. Phys. D: Appl. Phys. 33 R51

[2] Bogen P 1983 Proc. XVIth Int. Conf. on Phenomena in Ionized Gases ed W Böttcher et al (Düsseldorf: Institute for Theoretical Physics) pp 164-73

[3] Setser D W 1979 Reactive Intermediates in the Gas Phase (New York: Academic)

[4] Goehlich A, Kawetzki T and Döbele H F 1998 J. Chem. Phys. 1089362

[5] Loudon R 1983 The Quantum Theory of Light 2nd edn (Oxford: Clarendon)

[6] Pezé P, Paillous A, Siffre J and Dubreuil B 1993 J. Phys. D: Appl. Phys. 261622

[7] Paul P H 1994 J. Quant. Spectrosc. Radiat. Transfer 51511

[8] Dilecce G, Vigliotti M and De Benedictis S 2000 J. Phys. D: Appl. Phys. 33 L53

[9] Bittner J, Kohse-Höinghaus K, Meier U and Just Th 1988 Chem. Phys. Lett. 143571

[10] Preppernau B L, Pearce K, Tserepi A, Wurzberg E and Miller T A 1995 Chem. Phys. 196371

[11] Alekseev V and Setser D W 1996 J. Phys. Chem. 1005766

[12] Chang R S F, Horiguchi H and Setser D W 1980 J. Chem. Phys. $\mathbf{7 3} 778$

[13] Adams S F and Miller T A 1998 Chem. Phys. Lett. 295305

[14] Condon E U and Shortley G H 1970 The Theory of Atomic Spectra (New York: Cambridge University Press)

[15] Tung J H, Tang A Z, Salamo G J and Chan F T 1986 J. Opt. Soc. Am. B 3837

[16] Kielkopf J and Myneni K 1987 J. Phys. B: At. Mol. Phys. 20 2467

[17] van der Heijden H W P, Boogaarts M G H, Mazouffre S, van der Mullen J A M and Schram D C 2000 Phys. Rev. E 614402

[18] Gontier Y and Trahin M 1971 Phys. Lett. A 36463

[19] Pindzola M S 1978 Phys. Rev. A 171021

[20] Saxon R and Eichler J 1986 Phys. Rev. A 34199

[21] Omidvar K 1980 Phys. Rev. A 221576

[22] Bamford D J, Junsinski L E and Bischel W K 1986 Phys. Rev. A 34185

[23] Sabbagh J and Sadeghi N 1977 J. Quant. Spectrosc. Radiat. Transfer 17297

[24] Mazouffre S, Foissac C, Supiot P, Vankan P, Engeln R, Schram D C and Sadeghi N 2001 Plasma Sources Sci. Technol. 10168

[25] Mazouffre S, Engeln R, Vankan P and Schram D C 2001 Proc. Frontiers in Low Temperature Plasma Diagnostics IV ed W W Stoffels (Rolduc) pp 100-3

[26] Setser D W 2001 Proc. APP Spring Meeting on Diagnostics of Non-Equilibrium High Pressure Plasmas (Bad Honnef) pp 49-59, webpage http://www.ilp.physik.uniessen.de/doebele/Spring2001/index.html 